\title{
Review of: "Homocysteine-induced endoplasmic reticulum stress activates FGF21 via CREBH, resulting in browning and atrophy of white adipose tissue in Bhmt knockout mice."
}

Jibin Li

Potential competing interests: The author(s) declared that no potential competing interests exist.

In this study, the authors observed decreased body weight and fat mass in Bhmt KO mice model, and further investigated the possible role of Hcy-induced liver ER stress in adipose browning. The authors concluded that HCy-CREBH-FGF21-adipose browning pathway was responsible for the WAT atrophy in Bhmt KO mice. This finding is of great importance to understand the multiple roles of homocysteine in health and disease. However, there are a few concerns with this study:

1. Given that the plasma Hcy level of Bhmt KO mice is much higher ( $\sim 1$ fold) than that of WT mice, there is the possibility that HHcy may bring adverse effects on animal growth. Therefore, the body weights gaining of the animals in two groups should be shown to determine the effects of elevated Hcy on animal growth, rather than the final body weights be shown only.

2. Body weight gain is highly related to food intake. However in the present study, the authors didn't mention and compare the food intakes in two groups.

3. In the present study, authors observed elevated Hcy levels, ER stress pathways activation, increased FGF21 and iWAT browning in Bhmt KO model. Based on the above findings, the authors proposed that HCy-CREBH-FDF21-adipose browning pathway might drive this special phenotype. However, without further study design to verify this hypothesis, it is difficult to evaluate the causative effects of Hcyinduced ER stress in WAT browning. 